\title{
Double-eyelid Blepharoplasty-induced Scar Treated with a 10,600-nm Carbon Dioxide Fractional Laser
}

\author{
Young Koo Kim ${ }^{1}$ \\ Eun Soo Park ${ }^{2}$ \\ Sung Bin $\mathrm{Cho}^{3}$
}

\footnotetext{
${ }^{1}$ Yonsei Star Skin \& Laser Clinic, Seoul, Korea ${ }^{2}$ Department of Plastic and Reconstructive Surgery, College of Medicine, Soonchunhyang University, Bucheon, Korea

${ }^{3}$ Department of Dermatology and Cutaneous Biology Research Institute, Yonsei University College of Medicine, Seoul, Korea
}

Double-eyelid blepharoplasty has been performed frequently in Asian patients for cosmetic purposes. However, despite improvements in various modified surgical techniques, complications of asymmetry of double-eyelids, loosening or disappearance of the double fold, inclusion cyst, and scarring are common after double-eyelid blepharoplasty. In this report, we describe a patient whose double-eyelid blepharoplastyinduced scars were effectively treated using an ablative 10,600-nm carbon dioxide (C02) fractional laser. After four sessions of $\mathrm{CO} 2$ fractional laser treatment at one to two-month intervals, marked improvement of more than $80 \%$ was achieved, and the patient was satisfied with the results. In addition, possible side effects, particularly immediate or prolonged post-therapy erythema and unfolding of the double folds, were not observed. Accordingly, we suggest that $\mathrm{CO} 2$ fractional laser treatment be a safe and effective therapeutic option, other than surgical correction, for treatment for scar correction of double-eyelid blepharoplasty.

\section{Key words}

Carbon dioxide; Double-eyelid; Blepharoplasty; Fractional laser; Scar 


\section{INTRODUCTION}

Double-eyelid blepharoplasty has been performed a lot in Asian patients for cosmetic purposes. ${ }^{1-3}$ Accordingly, various incisional and non-incisional surgical techniques for creating double folds on the upper eyelids have been introduced to achieve better clinical outcomes and lessen the risk of side effects. ${ }^{1-3}$ However, despite improvements in these modified surgical techniques, complications of asymmetry of double-eyelids, loosening or disappearance of the double fold, inclusion cyst, and scarring are common after double-eyelid blepharoplasty. ${ }^{1,4}$ Especially, hypertrophic scars in medial canthal area after incisional double-eyelid blepharoplasty can be problems. Unnatural scars on the closed eye lids show and operation like lines look deep and bumpy. Asymmetry scars make doubleeyelid look asymmetric and cause eyelids to be puffy (sausage-like appearance).

Correction of the complications associated with blepharoplasty often requires an additional surgical procedure. ${ }^{4}$ However, surgical scar revision cannot be easily performed in some patients with a propensity of hypertrophic scars or keloids. In this report, we describe the case of a patient whose double-eyelid blepharoplasty-induced scars were effectively treated with an ablative 10,600-nm carbon dioxide (CO2) fractional laser.

\section{CASE REPORT}

A 26-year-old Korean woman with hypertrophic scars on the upper eyelids that had been present for six years was present at our clinic. She had undergone incisional double-fold blepharoplasty six years ago for cosmetic purposes. The hypertrophic scars were visible along the double-folds, and stood out when she closed her eyes. Especially, longer and more serious scar appeared on the left closed eyelid. Therefore, the left double-eyelid looked bigger and puffy when the eyes are open (Fig. 1A and B). However, she had never received a proper treatment for the surgical scars along the upper eyelids previously. She had no pertinent past medical or family history, including hypertrophic scars and keloids.

After obtaining informed consent, the patient was treated with four sessions of CO2 fractional laser treatment using an Ultrapulse Encore laser (Lumenis Inc., Santa Clara, CAl at one to two-month intervals. Prior to the laser treatment, the lesions were cleansed, and topical anesthetic cream containing 2.5\% lidocaine $\mathrm{HCl}$ and $2.5 \%$ prilocaine (EMLA cream; AstraZeneca $A B$, Södertälje, Sweden) was applied for one hour on the upper eyelids for local anesthesia. The laser fluence was initially delivered to the upper eyelid scars with the settings of $10 \mathrm{~mJ}$, a density of 1 ( $5 \%$ coverage), single pass, and $300 \mathrm{~Hz}$ using the Deep FX. mode. Immediately after the treatment, the lesions were cooled with ice packs. Prophylactic oral antibiotics were prescribed for three days, and she was instructed to apply a moisturizer.

One month after the first treatment, the scar tissue along the double folds had slightly improved. After all four sessions of $\mathrm{CO} 2$ fractional laser treatment, marked improvement of more than $80 \%$ was achieved, and she was satisfied with the results (Fig. 1C). Moreover, asymmetry and puffiness of the upper eyelids had improved. Post-therapy erythema spontaneously disappeared within two weeks. Otherwise, possible side effects, including oozing, bleeding, edema, post-therapy hyper- or hypopigmentation, prolonged post-therapy erythema, unfolding of double folds, and worsening of scars, were not observed (Fig. 1D).

\section{DISCUSSION}

C02 fractional lasers are used in the treatment of various dermatologic conditions, including various types of atrophic scars, syringomas, and inflammatory skin diseases, to deliver relatively high laser energy to target tissues with minimal side effects. ${ }^{5-9}$ Previous split-face study demonstrated that CO2 fractional
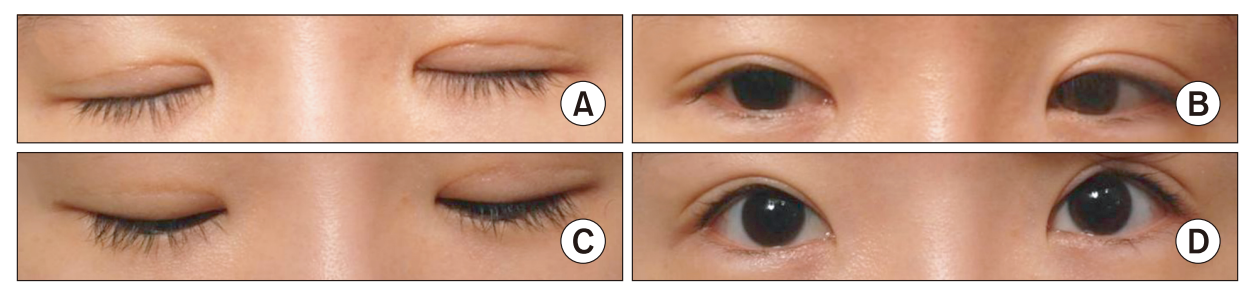

Fig. 1. (A and B) Hypertrophic surgical scars on the double folds with asymmetry and prolonged swelling of the upper eyelids. (C and D) Clinical improvement of surgical scars on the upper eyelids after four sessions of carbon dioxide fractional laser treatment at one to two-month intervals without remarkable side effects. 
laser treatments for acne scars and enlarged pores with higher-energy, lower-density settings were more effective than treatments with lower-energy, higherdensity laser settings. ${ }^{6}$ Another study also revealed that textural improvement was more effectively achieved by nonablative fractional laser treatment with laser settings of higher-energy and lower-density, which created deeper therapeutic columns. ${ }^{10}$

Additionally, combination of different laser settings for CO2 fractional lasers, namely Deep FX. and Active FX. modes, was suggested to raise the therapeutic efficacy of treatments for acne scars and enlarged pores. ${ }^{7}$ The Deep FX. mode, which was used in the present report, irradiates laser energy with a spot size of $0.12 \mathrm{~mm}$ that can penetrate the deep portions of target tissues. ${ }^{7}$ Comparatively, while the Active FX. mode provides a broader treatment zone with a spot size of $1.25 \mathrm{~mm}$, it is usually used to target skin lesions of the epidermaldermal junction or upper dermis. Thereby, combination therapy using these two modes in patients with acne scars and enlarged facial pores may provide more effective treatment, utilizing the broader treatment zone with the Active FX. mode and generation of deeper coagulation injury to deeply located scar tissue with the Deep FX. mode. ${ }^{7}$

In the present report, because the thickness of the skin along the upper eyelids is quite thin and because post-therapy wound care on lesions thereto is seriously limited by frequent eye movement, we decided to choose the laser settings of $10 \mathrm{~mJ}$, a density of 1 ( $5 \%$ coverage), single pass, and a spot size of $0.12 \mathrm{~mm}$. We believe that possible side effects, especially post-therapy erythema and unfolding of double folds, were avoided by using the laser settings of a small spot size and a shallow penetration depth. However, because a single C02 fractional laser treatment session can only irradiate a relatively small portion of the target tissue, with a percent coverage of less than $10 \%$, multiple treatment sessions were required to achieve satisfactory improvement.

In this report, we described the case of a patient with hypertrophic surgical scars of double folds on both upper eyelids that were effectively treated with $\mathrm{CO} 2$ fractional laser treatment using laser settings of a small spot size and a shallow penetration depth. We suggest that CO2 fractional laser treatment may be a safe and effective therapeutic option, other than surgical correction, in patients with complications from double-eyelid blepharoplasty. However, controlled, prospective studies should be conducted in larger populations to support our findings.

\section{REFERENCES}

1. Moon KC, Yoon ES, Lee JM. Modified double-eyelid blepharoplasty using the single-knot continuous buried non-incisional technique. Arch Plast Surg 2013;40:409-13.

2. Mikamo M. Mikamo's double-eyelid operation: the advent of Japanese aesthetic surgery. 1896. Plast Reconstr Surg 1997; 99:664; discussion 664-9.

3. Ko RY, Baek RM, Oh KS, Lim JH. Complication of non-incision oriental blepharoplasty: is disappearance of the lid crease a fearful complication? J Korean Soc Plast Reconstr Surg 2000; 27:199-203.

4. Zhang Y, Yuan L, Sun B, Jin R, Liu T, Wang X, et al. Repair of unsatisfactory double eyelid after double-eyelid blepharoplasty in Asian patients. Arch Facial Plast Surg 2010;12:236-40.

5. Chapas AM, Brightman L, Sukal S, Hale E, Daniel D, Bernstein $L J$, et al. Successful treatment of acneiform scarring with CO2 ablative fractional resurfacing. Lasers Surg Med 2008;40:381-6.

6. Jung JY, Lee JH, Ryu DJ, Lee SJ, Bang D, Cho SB. Lowerfluence, higher-density versus higher-fluence, lower-density treatment with a 10,600-nm carbon dioxide fractional laser system: a split-face, evaluator-blinded study. Dermatol Surg 2010;36:2022-9.

7. Cho SB, Lee SJ, Kang JM, Kim YK, Chung WS, Oh SH. The efficacy and safety of 10,600-nm carbon dioxide fractional laser for acne scars in Asian patients. Dermatol Surg 2009;35:195561.

8. Cho SB, Kim HJ, Noh S, Lee SJ, Kim YK, Lee JH. Treatment of syringoma using an ablative 10,600-nm carbon dioxide fractional laser: a prospective analysis of 35 patients. Dermatol Surg 2011;37:433-8.

9. Park J, Choi MJ, Goo B, Cho SB. Refractory pyoderma gangrenosum effectively treated using a 10,600-nm carbon dioxide fractional laser. Dermatol Surg 2013;39:477-9.

10. Walgrave S, Zelickson B, Childs J, Altshuler G, Erofeev A, Yaroslavsky I, et al. Pilot investigation of the correlation between histological and clinical effects of infrared fractional resurfacing lasers. Dermatol Surg 2008;34:1443-53. 